\title{
INFLUÊNCIA DE CONDIÇÕES SOCIOECONÔMICAS E DE SAÚDE EM CRIANÇAS NA OCORRÊNCIA DE DIARREIA INFANTIL
}

\section{INFLUENCE OF SOCIOECONOMIC AND HEALTH CONDITIONS IN CHILDREN IN THE OCCURRENCE OF CHILDHOOD DIARRHEA}

\section{INFLUENCIA DE CONDICIONES SOCIOECONÓMICAS Y DE SALUD EN NIÑOS EN LA OCURRENCIA DE DIARREIA INFANTIL}

\author{
Emanuella Silva Joventino ${ }^{1}$, Brena Shellem Bessa Oliveira ${ }^{2}$, Rhaiany Kelly Lopes Oliveira ${ }^{3}$, \\ Francisca Mayra Sousa Melo ${ }^{4}$, Mônica Oliveira Batista Oriá ${ }^{5}$, Lorena Barbosa Ximenes ${ }^{6}$
}

\begin{abstract}
RESUMO
Objetivo: Verificar a associação entre diarreia infantil com características socioeconômicas e condições de saúde dessas crianças. Método: Estudo transversal realizado com 448 mães de crianças menores de cinco anos, cujos filhos encontravam-se cadastrados em seis Unidades de Atenção Primária à Saúde do interior do Ceará. Os dados foram analisados por meio dos testes linear by linear e quiquadrado. Resultados: Verificou-se associação estatisticamente significante entre diarreia infantil e as seguintes variáveis: menor renda per capita $(\mathrm{p}=0,001)$; idade da criança maior que 36 meses $(\mathrm{p}=0,001)$; inexistência de sabão próximo às torneiras da casa $(\mathrm{p}=0,018)$; não realizar tratamento da água $(\mathrm{p}=0,012)$; origem da água $(\mathrm{p}=0,010)$; não manter cobertos os recipientes de armazenamento da água $(\mathrm{p}=0,019)$; possuir sanitário sem descarga $(\mathrm{p}=0,046)$; e criança ter histórico de internação no primeiro mês de vida $(\mathrm{p}=0,049)$. Conclusão: Constatou-se que a renda e que algumas variáveis referentes à condições higiênico-sanitárias e condições de saúde da criança estiveram associadas à ocorrência de diarreia infantil.
\end{abstract}

Descritores: Diarreia infantil; Saúde da criança; Enfermagem.

\begin{abstract}
Objective: To verify the association between childhood diarrhea with socioeconomic characteristics and health conditions of these children. Methods: A cross-sectional study was performed with 448 mothers of children under five years of age, whose children were enrolled in six Primary Health Care Units in the interior of Ceará. Data were analyzed by means of linear and chi-square tests. Results: There was a statistically significant association between infant diarrhea and the following variables: lower per capita income $(\mathrm{p}=0.001)$; lower child age $(\mathrm{p}=0.001)$; not having soap near the taps of the house $(\mathrm{p}=0.018)$; not to perform water treatment $(\mathrm{p}=0.012)$; water source $(\mathrm{p}=0.010)$; not keeping water storage containers covered $(\mathrm{p}=0.019)$; toilet without flush $(\mathrm{p}=0.046)$; and the child had a history of hospitalization in the

\footnotetext{
${ }^{1}$ Doutora em Enfermagem. Professora da Universidade da Integração Internacional da Lusofonia Afro-Brasileira (UNILAB)

${ }^{2}$ Enfermeira. Mestranda em Enfermagem na UNILAB e bolsista CAPES

${ }^{3}$ Enfermeira. Mestranda em Enfermagem na UNILAB.

${ }^{4}$ Enfermeira. Mestre em Enfermagem e Professora da Faculdade Princesa do Oeste (FPO).

${ }^{5}$ Doutora em Enfermagem e Professora da Universidade Federal do Ceará.

${ }^{6}$ Doutora em Enfermagem e Professora da Universidade Federal do Ceará.
} 
first month of life $(p=0.049)$. Conclusion: It was verified that the income and that some variables referring to hygienic-sanitary conditions and health conditions of the child are associated with the occurrence of infantile diarrhea.

Descriptors: Diarrhea, Infantile; Child health; Nursing.

\section{RESUMEN}

Objetivo: Verificar la asociación entre diarrea infantil con características socioeconómicas y condiciones de salud de esos niños. Método: Estudio transversal realizado con 448 madres de niños menores de cinco años, cuyos hijos se encontraban catastrados en seis Unidades de Atención Primaria a la Salud del interior de Ceará. Los datos fueron analizados por medio de las pruebas lineales lineal y cuadrado. Resultados: Se verificó asociación estadísticamente significante entre diarrea infantil y las siguientes variables: menor ingreso per cápita $(\mathrm{p}=$ $0,001)$; menor edad del niño $(\mathrm{p}=0,001)$; no tener jabón cerca de los grifos de la casa $(\mathrm{p}=$ $0,018)$; no realizar tratamiento del agua $(\mathrm{p}=0,012)$; origen del agua $(\mathrm{p}=0,010)$; no mantener cubiertos los recipientes de almacenamiento del agua $(\mathrm{p}=0,019)$; salud no tiene ninguna liberación ( $\mathrm{p}=0,046)$; no realizar lactancia exclusiva hasta los seis meses $(\mathrm{p}=0,005)$ y el niño tiene antecedentes de internación en el primer mes de vida $(\mathrm{p}=0,049)$. Conclusión: Se constató que la renta y que algunas variables referentes a condiciones higiénico-sanitarias y condiciones de salud del niño están asociadas a la ocurrencia de diarrea infantil.

Descritores: Diarrea Infantil; Salud del Niño; Enfermería.

\section{INTRODUÇÃO}

Nos últimos 25 anos, a mortalidade infantil no mundo caiu mais da metade, de 12,7 milhões em 1990 para 5,9 milhões em 2015. Contudo, ainda morrem cerca de 16 mil crianças por dia, sendo a diarreia, pneumonia, prematuridade, malária e complicações no parto as principais causas desses óbitos. ${ }^{1}$

O Brasil reduziu em $73 \%$ a sua taxa de mortalidade infantil. ${ }^{1}$ A redução também foi alcançada em esferas regionais; porém, ainda são notórias disparidades, uma vez que as crianças que residem no norte e nordeste apresentam maior risco de morrerem por diarreia quando comparadas com as crianças que vivem nas demais regiões. $^{2}$

Além disso, ressalta-se que a maioria das mortes infantis poderia ser evitada, uma vez que a diarreia é um agravo sensível à atenção primária, sendo passível de ser prevenida, caso algumas condutas sejam tomadas, tais como: medidas básicas de higiene, ampliação da rede vacinal, acesso ao saneamento básico, tratamento público da água destinada ao consumo humano e promoção do aleitamento materno exclusivo até os seis meses de idade da criança. ${ }^{3}$

Entretanto, mesmo sabendo da relação causa e efeito entre esses fatores e a diarreia, percebe-se que esse agravo 
ainda se faz presente. Assim, faz-se necessária uma investigação do contexto no qual a diarreia se apresenta, a fim de esclarecer os motivos que ainda levam à ocorrência dessa patologia, visto que já foram referidas formas de prevenção na literatura.

Ademais, os fatores de risco associados à doença diarreica aguda podem ser explicados dentro de um modelo multicausal, incluindo aspectos socioeconômicos, políticos, demográficos, sanitários, ambientais e culturais. Dessa forma, nos países em desenvolvimento, como o Brasil, a incidência dessa patologia está diretamente relacionada à ineficiência dos serviços de saneamento básico e às condições sociodemográficas precárias nas quais a população infantil encontra-se inserida. $^{4}$

Diante disso, faz-se premente investigar as associações entre o perfil sociodemográfico, sanitário e de condições de saúde de crianças com a ocorrência de diarreia infantil, tendo em vista que a assistência à saúde deve enfocar, sobretudo, a prevenção de doenças e agravos, bem como na promoção da saúde.

Dessa maneira, ao avaliar os fatores associados à diarreia o enfermeiro poderá direcionar seus cuidados, intervenções, orientações e elaborar estratégias de educação em saúde, com condutas específicas para as famílias que estiverem mais expostas a fatores de risco.

Assim, o objetivo deste estudo foi verificar a associação entre ocorrência de diarreia infantil com características socioeconômicas e com condições de saúde dessas crianças.

\section{METODOLOGIA}

Estudo transversal, quantitativo, realizado com mães de crianças menores de cinco anos cujos filhos encontravam-se cadastrados em uma das seis Unidades de Atenção Primária à Saúde (UAPS), do interior do Ceará, selecionadas pelo estudo. Optou-se por incluir mães de crianças com menos de cinco anos, visto que a diarreia infantil é mais prevalente nessa faixa etária.

A partir da fórmula para o cálculo de populações finitas, obteve-se uma amostra ideal de 448 mães. A coleta de dados ocorreu nas próprias UAPS entre os meses de novembro de 2009 e fevereiro de 2010, por meio de entrevista, utilizando-se um formulário semiestruturado que abordava questões sociodemográficas, sanitárias e de saúde da criança. 
Como critérios de inclusão, adotaram-se: mães com pelo menos um filho com idade inferior a cinco anos; e mães cujos filhos fossem acompanhados nas UAPS selecionados pelo estudo. O critério de exclusão seguido foi mães com problemas mentais que impossibilitassem a fidedignidade das respostas obtidas. Enfatiza-se que nas tabelas ocorreu a variação do número de participantes respondentes em cada variável, pois alguns questionamentos não foram respondidos por toda a amostra, já que algumas perguntas somente eram feitas caso a participante desse uma resposta afirmativa no questionamento anterior.

Os dados foram organizados e analisados através do programa IBM SPSS Statistics (versão 20). Para a análise comparativa foram utilizados os testes linear by linear e teste quiquadrado, estabelecendo-se nível de significância inferior a 0,05 .

O estudo foi aprovado pelo Comitê de Ética em Pesquisa da Universidade Federal do Ceará, sob protocolo $n^{\circ}$ 92/09, sendo respeitadas todas as normas relacionadas a pesquisas envolvendo seres humanos.

\section{RESULTADOS}

De acordo com a Tabela 1, verificouse associação estatisticamente significante entre episódio anterior de diarreia infantil e as seguintes variáveis: renda per capita inferior a um salário mínimo $(\mathrm{p}=0,001)$ e idade da criança superior a 36 meses $(\mathrm{p}=0,001)$.

Tabela 1 - Associação entre as condições socioeconômicas familiares e a ocorrência de episódios anteriores de diarreia infantil. Fortaleza, Ceará.

\begin{tabular}{|c|c|c|c|}
\hline \multirow[t]{3}{*}{ Variáveis } & \multicolumn{2}{|c|}{ Episódio Anterior de Diarreia } & \multirow[t]{3}{*}{$p$} \\
\hline & Sim & Não & \\
\hline & $\mathrm{N}-\%$ & $\mathrm{~N}-\%$ & \\
\hline \multicolumn{4}{|c|}{ Faixa Etária da Mãe $(\mathrm{n}=446)$} \\
\hline$\leq 18$ anos & $21-63,6$ & $12-36,4$ & \multirow{4}{*}{$0,623^{\mathrm{a}}$} \\
\hline $19-29$ anos & $134-55,8$ & $106-44,2$ & \\
\hline $30-39$ anos & $82-60,3$ & $54-39,7$ & \\
\hline$\geq 40$ anos & $23-62,2$ & $14-37,8$ & \\
\hline \multicolumn{4}{|c|}{ Estado Civil da Mãe $(n=444)$} \\
\hline Com companheiro & $216-58,1$ & $156-41,9$ & \multirow[t]{2}{*}{$0,631^{b}$} \\
\hline Sem companheiro & $44-61,1$ & $28-38,9$ & \\
\hline \multicolumn{4}{|c|}{ Escolaridade da Mãe (n=425) } \\
\hline$<7$ anos & $61-63,5$ & $35-36,5$ & \multirow[t]{3}{*}{$0,102^{\mathrm{a}}$} \\
\hline $7-10$ anos & $106-60,2$ & $70-39,8$ & \\
\hline$>10$ anos & $92-53,8$ & $79-46,2$ & \\
\hline
\end{tabular}




\begin{tabular}{|c|c|c|c|}
\hline$\leq 4$ & $163-58,2$ & $117-41,8$ & $0,995^{\mathrm{a}}$ \\
\hline$\overline{5}-6$ & $66-59,5$ & $45-40,5$ & \\
\hline$\geq 7$ & $32-57,1$ & $24-42,9$ & \\
\hline \multicolumn{4}{|c|}{ Renda per capita $(S M) *(n=413)$} \\
\hline $0-1 / 4 \mathrm{SM}$ & $40-69$ & $18-31$ & \multirow{4}{*}{$\mathbf{0 , 0 0 1}{ }^{\mathrm{a}}$} \\
\hline $1 / 4-1 / 2 \mathrm{SM}$ & $83-61$ & $53-39$ & \\
\hline $1 / 2-1 \mathrm{SM}$ & $93-62,4$ & $56-37,6$ & \\
\hline$\geq 1$ & $26-37,1$ & $44-62,9$ & \\
\hline \multicolumn{4}{|c|}{ 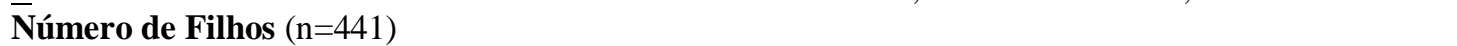 } \\
\hline$\geq 3$ & $231-56,5$ & $164-43,5$ & \multirow[t]{2}{*}{$0,799^{\mathrm{b}}$} \\
\hline$\leq 4$ & $26-58,3$ & $20-41,7$ & \\
\hline \multicolumn{4}{|c|}{$\overline{\text { Sexo da Criança }(n=447)}$} \\
\hline Masculino & $133-59,9$ & $89-40,1$ & \multirow[t]{2}{*}{$0,517^{\mathrm{b}}$} \\
\hline Feminino & $128-56,9$ & $97-43,1$ & \\
\hline \multicolumn{4}{|c|}{ Idade da criança $(n=447)$} \\
\hline $0-6$ meses & $18-20,7$ & $68-78,2$ & \multirow[t]{4}{*}{$<0,001^{a}$} \\
\hline 7 - 12 meses & $29-53,7$ & $25-46,3$ & \\
\hline 13 - 36 meses & $94-65,3$ & $50-34,7$ & \\
\hline$>36$ meses & $120-73,6$ & $43-26,4$ & \\
\hline
\end{tabular}

*SM: Salário mínimo. O valor do SM durante o estudo era de R\$510,00.

a - linear by linear

$\mathrm{b}-$ chi square

Ao se analisar as situações da casa $(\mathrm{p}=0,018)$; realização de habitacionais das famílias entrevistadas, tratamento domiciliar na água que a buscou-se levar em conta que aspectos criança consome $(\mathrm{p}=0,012)$; ao fato de a encontrados dentro de casa poderiam ser criança ingerir água de outras fontes que fatores de risco para a ocorrência da não a mineral, como água de cisterna, lago, diarreia infantil. Dessa forma, conforme chuva, entre outras $(p=0,010)$; cobertura mostra a Tabela 2, identificaram-se dos recipientes de armazenamento da água associações estatisticamente significantes $\quad(p=0,019)$ e sanitário da casa sem descarga entre a ocorrência de diarreia infantil e a $\quad(p=0,046)$. inexistência de sabão próximo às torneiras

Tabela 2 - Associação entre as condições de moradia e hábitos familiares com a ocorrência de episódios anteriores de diarreia infantil. Fortaleza-CE, 2010.

\begin{tabular}{lccc}
\hline Variáveis & \multicolumn{2}{c}{ Episódio anterior de diarreia } & $P$ \\
& Sim & Não & \\
& $\mathrm{N}-\%$ & $\mathrm{~N}-\%$ \\
\cline { 2 - 3 } $\begin{array}{l}\text { Sabão próximo às torneiras para lavar as mãos } \\
(\mathrm{n}=428)\end{array}$ & & & \\
Sim & $230-58,5$ & $163-41,5$ & $\mathbf{0 , 0 1 8}^{\mathbf{a}}$ \\
Não & $25-71,4$ & $10-28,6$ & \\
Tratamento domiciliar da água que a criança & & &
\end{tabular}


consome $(n=432)$

Sim

Não

Destino do Lixo da Residência (n= 447)

Coleta Pública

Jogado a céu aberto/rio

Origem da água consumida pela criança $(n=$ 441)

Mineral

Outros

Cobertura dos recipientes de água consumida $(n=413)$

Cobertos

Descobertos

Não se aplica

Tipo de sanitário $(n=444)$

Com descarga d'água

Sem descarga d'água

$\mathrm{a}$ - linear by linear

$\mathrm{b}$ - chi square

Pode-se observar na amostra

estudada, por meio da Tabela 3 , que houve

relação significante entre a ocorrência

anterior de diarreia e as seguintes

$\begin{array}{ccc}86-67,2 & 42-32,8 & \mathbf{0 , 0 1 2}^{\mathbf{a}} \\ 170-55,9 & 134-44,1 & \\ & & \\ 247-57,7 & 181-42,3 & 0,167^{\mathrm{b}} \\ 14-73,7 & 5-26,3 & \end{array}$

$165-54,8 \quad 136-45,2$

$\mathbf{0 , 0 1 0}$

$95-67,9 \quad 45-32,1$

$182-61,9 \quad 112-38,1$

$\begin{array}{ll}33-52,4 & 30-47,6 \\ 24-42,9 & 32-57,1\end{array}$

$0,019^{\mathrm{a}}$

$190-55,6 \quad 152-44,4$

$68-66,7 \quad 34-33,3$

$0,046^{\mathrm{b}}$

Tabela 3 - Associação entre as condições de saúde da criança e a ocorrência de episódios anteriores de diarreia infantil. Fortaleza, Ceará.

\begin{tabular}{|c|c|c|c|}
\hline \multirow[t]{3}{*}{ Variáveis } & \multicolumn{2}{|c|}{ Episódio anterior de diarreia } & \multirow[t]{3}{*}{$p$} \\
\hline & Sim & Não & \\
\hline & $\mathrm{N}-\%$ & $\mathrm{~N}-\%$ & \\
\hline \multicolumn{4}{|l|}{ Peso ao nascer $(n=410)$} \\
\hline Baixo peso $(>2500 \mathrm{~g})$ & $22-50,0$ & $22-50,0$ & $0,420^{\mathrm{a}}$ \\
\hline Insuficiente $(2500$ - 2999g) & $30-54,5$ & $25-45,5$ & \\
\hline Adequado (3000 - 3999g) & $161-61,2$ & $102-38,8$ & \\
\hline Excesso de peso $(<4000 \mathrm{~g})$ & $26-54,2$ & $22-45,8$ & \\
\hline \multicolumn{4}{|c|}{$\begin{array}{l}\text { Existência de doenças na criança atualmente } \\
(n=447)\end{array}$} \\
\hline Sim & $62-76,5$ & $19-23,5$ & $<\mathbf{0 , 0 0 1}{ }^{\text {b }}$ \\
\hline Não & $199-54,4$ & $167-45,6$ & \\
\hline \multicolumn{4}{|c|}{$\begin{array}{l}\text { Uso de mamadeira para alimentação da } \\
\text { criança }(n=447)\end{array}$} \\
\hline Sim & $138-59,2$ & $95-40,8$ & $0,708^{\mathrm{b}}$ \\
\hline Não & $123-57,5$ & $91-42,5$ & \\
\hline \multicolumn{4}{|c|}{$\begin{array}{l}\text { Internação da criança no primeiro mês de vida } \\
(\mathrm{n}=447)\end{array}$} \\
\hline $\operatorname{Sim}$ & $52-68,4$ & $24-31,6$ & $\mathbf{0 , 0 4 9} 9^{\mathrm{b}}$ \\
\hline Não & $209-56,3$ & $162-43,7$ & \\
\hline
\end{tabular}


a - linear by linear

$\mathrm{b}$ - chi square

\section{DISCUSSÃO}

Apesar dos achados do presente estudo não terem evidenciado associação entre diarreia infantil e algumas variáveis sociodemográficas, reconhece-se a possibilidade da faixa etária materna influenciar a ocorrência desta patologia, pois, acredita-se que mães mais jovens têm pouca experiência para identificar os sinais e sintomas de patologias e agravos infantis, bem como podem ter habilidades ainda insuficientes para promover os cuidados essenciais aos filhos. ${ }^{5}$ Destaca-se também a influência da escolaridade materna nos cuidados prestados a criança, por ser um marcador da condição socioeconômica da mãe e de sua família, relacionando-se nesse contexto com o perfil cultural e comportamental. $^{3}$

Quanto à renda per capita, autores mostraram que esse determinante social está diretamente relacionado ao desenvolvimento de enteroparositoses e diarreia infantil ${ }^{2}$, bem como interfere na autoeficácia materna para prevenir esta patologia. ${ }^{6}$ Esses achados corroboram os resultados do presente estudo.
Além deste fato, acredita-se que a renda insuficiente pode favorecer a ocorrência de condições sociais precárias e um menor nível de esclarecimento dos pais sobre as medidas preventivas de diarreia, o que interfere diretamente na capacidade de assegurar uma boa alimentação para as crianças e, consequentemente, prevenir e tratar diarreia. $^{7}$

Em alguns lugares, a falta de entendimento do que realmente seja diarreia e de que ela é uma doença grave pode impedir um avanço mais significativo no seu combate. Isso foi demonstrado por estudo realizado na África, que constatou a eficiência de uma campanha na mídia informando a respeito das formas de contaminação, de reconhecimento e de percepção da gravidade de diarreia, além de incentivar o uso de Soluções de Reidratação Oral (SRO) como forma de combater a desidratação, principal complicação da diarreia infantil. Salientase que o uso de SRO entre os cuidadores teve aumento de $10 \%$ após a campanha. ${ }^{8}$ Assim, fica evidente a importância de se investir em educação em saúde a fim de orientar a população acerca desse tema, o 
que poderá contribuir para a redução das taxas de diarreia infantil.

A idade da criança também se destacou como um importante preditor no desenvolvimento de diarreia infantil. Entretanto, neste estudo os episódios diarreicos ocorreram com maior frequência em crianças maiores de 36 meses, o que se contrapõe a pesquisas realizadas em Moçambique, que demonstrou que essa patologia é mais recorrente em crianças menores de um ano. ${ }^{9}$

Outro fator que demonstrou associação significativa com a ocorrência de diarreia foi a ausência de sabão próximo às torneiras $(71,4 \%)$. A relevância de ter esse produto de higiene também foi ressaltada por pesquisa realizada no Quênia, a qual concluiu que o índice de prevalência de diarreia em crianças menores cinco anos caiu $41 \%$ em residências que possuíam sabão para fazer a higienização das mãos, quando comparadas com residências que não possuíam esse produto de limpeza. ${ }^{10} \mathrm{~A}$ existência de sabão próximo às torneiras da casa proporciona uma maior praticidade no hábito de se lavar as mãos; além disso, sabe-se que investir em melhoria dos hábitos de higiene, principalmente na idade pré-escolar é uma intervenção eficaz, pois possibilita um melhor controle de doenças infectocontagiosas. ${ }^{11}$

Porém, ainda acerca dessa variável, foi possível notar que a associação entre ter sabão próximo às torneiras e acontecer o desenvolvimento de diarreia infantil também foi considerável. Esse resultado pode estar associado à higienização das mãos de forma inadequada pelos cuidadores e pela criança. Outro fator que pode ter tido influência nesse resultado se refere à ocorrência de episódios diarreicos anteriores, a qual pode ter favorecido o uso deste produto de limpeza, a fim de reduzir novos casos dessa patologia.

Além disso, crianças apresentam maior probabilidade de serem acometidas por doenças transmitidas através de água e comida, devido à imaturidade do sistema digestivo e da ainda ineficiente barreira imunológica. ${ }^{9}$ Assim sendo, torna-se essencial a realização de tratamentos adequados da água para consumo humano, uma vez que esta pode veicular microorganismos e propiciar o desenvolvimento destes em seres humanos. ${ }^{12}$

Todavia, neste estudo notou-se associação significante entre a realização de tratamentos na água (filtrar, ferver, coar através de um pano) no domicílio e a 
ocorrência de diarreia infantil. Esse achado pode não estar diretamente associado ao ato de realizar o tratamento na água, mas sim no local onde ela é armazenada, o qual pode conter microorganismos e resíduos que podem estar contaminando-a e, consequentemente, favorecendo o desenvolvimento dessa afecção.

Ressalta-se que o armazenamento correto da água, bem como a cobertura adequada dos recipientes evitam a sua exposição a insetos vetores e poeira, o que poderia poluir o líquido de consumo contínuo. ${ }^{13}$ Percebeu-se que, neste estudo, a diarreia esteve associada estatisticamente com a cobertura dos recipiente onde a água era armazenada. Esse resultado pode estar relacionado à cobertura não efetiva, o que pode ter possibilitado a contaminação da água e ocasionado diarreia nas crianças.

Ainda, levando em consideração as características de moradia das famílias que fizeram parte da pesquisa, a presença de vaso sanitário com descarga mostrou-se influente na prevenção do adoecimento das crianças. Corroborando este achado, estudo realizado em Macapá mostrou associação significativa entre o desenvolvimento da diarreia infantil e a ausência de vaso sanitário na residência. ${ }^{14}$
A preocupação com a melhoria do saneamento básico existe em praticamente todos os países, em especial os que ainda estão em processo de desenvolvimento, uma vez que condições sanitárias desfavoráveis estão diretamente relacionadas com o desenvolvimento de diarreia infantil. ${ }^{15}$

O número de internações de crianças menores de cinco anos pode ser usado como parâmetro para o desenvolvimento de estratégias no setor de saúde coletiva, na prevenção de doenças e na educação para a saúde das famílias, tendo em vista que vários são os fatores de risco para que as internações aconteçam em crianças de pouca idade.

Nesta pesquisa, apesar de a utilização de mamadeira para alimentação da criança não ter se relacionado com a ocorrência de diarreia, sabe-se da influência dessa prática no desmame precoce, bem como, na contaminação de crianças, muitas vezes devido à má higienização deste recurso e manutenção inadequada de utensílios; além de prejudicar no desenvolvimento da musculatura oral da criança, dificultam a sucção e a mastigação. ${ }^{16,17}$

Além disso, destaca-se o fato de que, se as intervenções e pacotes de cuidado 
comprovadamente eficazes fossem implementados, seria reduzido $54 \%$ dos casos de diarreia em 2025 a um custo de $\$ 3,8$ bilhões, menos do que se gasta com hospitalizações e tratamentos para esta doença. Assim, várias intervenções poderiam reduzir a morbidade e a gravidade global da diarreia, com possíveis benefícios para o desenvolvimento infantil e dos países. ${ }^{4}$

\section{CONCLUSÃO}

Percebe-se que inúmeros fatores podem ser decisivos no acometimento por uma doença gastrintestinal ou de outra origem, capaz de causar diarreia numa criança. Mais precisamente, conforme identificado neste estudo, a baixa renda da família, idade da criança superior a 36 meses, o cuidado na origem da água que a criança ingere, o despejo inadequado dos dejetos do domicílio, internações durante o primeiro mês de vida e o acometimento de doenças, atualmente, estiveram explicitamente relacionados com os episódios de diarreia em algum momento da vida. Conhecendo esses fatores, pode-se agir de forma mais direcionada na busca pela prevenção de tal agravo à saúde infantil.

Cabe à equipe de enfermagem agir sobre essa questão, bem como aos demais profissionais de saúde; além das autoridades competentes no que diz respeito às obras de saneamento básico e tratamento de água, com ações que garantam a segurança à saúde da população que usufrui do serviço público. O Enfermeiro, principal responsável pelas atividades de Educação em Saúde e profissional de grande atuação na Atenção Básica, precisa estar mais próximo dos dados epidemiológicos da sua população, para, assim, compreender em quais aspectos deverá intensificar ações preventivas. Também, necessita buscar minimizar os fatores de risco para a diarreia infantil.

Por fim, ressalta-se como fatores limitantes o fato deste estudo ter sido unicêntrico e de ter utilizado a amostragem por conveniência. Dessa forma, destaca-se a necessidade de serem realizados estudos que promovam o acompanhamento mais detalhado acerca deste tema.

Group; United Nations. Levels \& trands in child mortality: report 2015: estimates

\section{REFERÊNCIAS}

1. United Nations Children's Fund; World Health Organization; World Bank 
developed by the UN Inter-agency group for child mortality estimation. New York: UNICEF; 2015 [citado em 02 maio 2018]. 36 p. Disponível em: http://www.unicef.org/publications/files/C hild_Mortality_Report_2015_Web_8_Sept -15.pdf

2. Bühler HF, Ignotti E, Neves SMAS, Hacon SS. Análise espacial de indicadores integrados determinantes da mortalidade por diarreia aguda em crianças menores de 1 ano em regiões geográficas. Cad Saúde Pública [Internet]. 2014 [citado em 02 maio 2018]; 19(10):4131-40. doi: http://dx.doi.org/10.1590/1413812320141910.09282014 3. Imada KS, Araújo TS, Muniz PT, Pádua VL. Socioeconomic, hygienic, and sanitation factors in reducing diarrhea in the Amazon. Rev Saúde Pública [Internet]. 2016 [citado em 02 maio 2018]; 50:77. doi: http://dx.doi.org/10.1590/s15188787.2016050006505

4. Bühler HF, Ignotti E, Neves SMAS, Hacon SS. Análise espacial de indicadores integrados de saúde e ambiente para morbimortalidade por diarreia infantil no Brasil, 2010. Cad Saúde Pública [Internet]. 2014 [citado em 02 maio 2018]; 30(9):1921-34. doi:

http://dx.doi.org/10.1590/0102311X00078013

5. Joventino ES, Freitas LV, Vieira NFC, Aquino PS, Pinheiro AKB, Ximenes LB . Habilidades maternas para prevenção e manejo da diarreia infantil. Cien Enferm. [Internet]. 2013 [citado em 02 maio 2018]; 19(2):67-76. Disponível em: http://dx.doi.org/10.4067/S071795532013000200007

6. Oliveira RKL, Oliveira BSB, Colares JB, Silva MJN, Melo FMS, Joventino ES. Influence of socio-economic conditions and maternal knowledge in selfeffectiveness for prevention of childhood diarrhea. Esc Anna Nery Rev Enferm.
[Internet]. 2017 [citado em 05 nov 2017]; 21(4):e20160361. doi: http://dx.doi.org/10.1590/2177-9465-ean2016-0361

7. Vasconcelos MJOB, Rissin A, Figueiroa JN, Lira PIC, Batista Filho M. Factors associated with diarrhea in children under five years old in the state of Pernambuco, according to surveys conducted in 1997 and 2006. Rev Saúde Pública [Internet]. 2018 [citado em 02 maio 2018]; 52:48. doi: https://doi.org/10.11606/S15188787.2018052016094

8. Kassegne S, Kays MB, Nzohabonayo J. Evaluation of a social marketing intervention promoting oral rehydration salts in Burundi. BMC Public Health [Internet]. 2011 [citado em 02 maio 2018]; 11:155. doi: https://doi.org/10.1186/1471-2458-11-155 9. Nampossa T, Sigaúque B, Machevo S, Macete E, Alonso P, Bassat Q, et al. Severe malnutrition among cildren under te age of 5 yerars admitted to a rural district hospital in Southern Mozambique. Public Health Nutr. [Internet]. 2013 [citado em 02 maio 2018];16(9):1565-74. doi: https://doi.org/10.1017/S13689800130010 80

10. Baker KK, Dil FF, Ferdous F, Ahmed S, Kumar DS, Faruque AS, et al. Association between moderate-to-severe diarrhea in young children in the Global Enteric Multicenter Study (GEMS) and types of handwashing materials used by caretakers in Mirzapur, Bangladesh. Am J Trop Med Hyg. [Internet]. 2014 [citado em 02 maio 2018]; 91(1):181-89. doi: https://doi.org/10.4269/ajtmh.13-0509 11. Silva AVM, Corrêa BS, Carneiro IM, Pinto PR, Almeida AAP, Silva JH. Ações de extensão para promoção da saúde em creche no município de Belo Horizonte, MG. Interagir: pensando a extensão [Internet]. 2016 [citado em 02 
maio 2018]; (22):32-45. Disponível em: https://www.e-

publicacoes.uerj.br/index.php/interagir/arti cle/view/17548/20117

12. Nunes SS, Soares FMP, Reis JS. Análise bacteriológica da água de reservatórios domiciliares do município de Coari - Amazonas. SaBios: Rev Saúde Biol. [Internet]. 2015 [citado em 02 maio 2018]; 10(3):09-14. Disponível em: http://revista2.grupointegrado.br/revista/in dex.php/sabios2/article/view/1265

13. Rego AP, Lima SP, Costa MCMDR, Santos LMC, Medeiro WR, Cavalcante ES. Conhecimento das mães de crianças internadas em um hospital universitário acerca da diarreia. Rev Rene [Internet]. 2014 [citado em 02 maio 2018]; 15(1):29-36. Disponível em:

http://www.periodicos.ufc.br/index.php/ren e/article/viewFile/3074/2361.

14. Porcy C, Ferro TAF, Monteiro SG, Menezes RAO, Barbosa FHF, Monteiro Neto V. Epidemiologia da doença diarreica associada às Escherichia coli diarreiogênicas em crianças residentes em uma área alagada de Macapá - Amapá, Brasil. Ciênc Equatorial [Internet]. 2013 [citado em 02 maio 2018]; 3(1):57-68.

Disponível em:

https://periodicos.unifap.br/index.php/cien ciaequatorial/article/view/804

15. Azage M, Kumie A, Worku A, Bagtzoglou AC. Childhood diarrhea in high and low-level districts in the Amhara region, northwest Ethiopia: a multilevel modeling. J Health Popul Nutr. [Internet]. 2016 [citado em 02 maio 2018]; 35(13):114. Disponível em:

https://jhpn.biomedcentral.com/articles/10. 1186/s41043-016-0052-2

16. Pellegrinelli ALR, Pereira SCL, Ribeiro IP, Santos LC. Influência do uso de chupeta e mamadeira no aleitamento materno exclusivo entre mães atendidas em um Banco de Leite Humano. Rev Nutr. [Internet]. 2015 [citado em 02 maio 2018]; 28(6): 631-39. doi:

http://dx.doi.org/10.1590/141552732015000600006

17. Bhutta ZA1, Das JK, Walker N, Rizvi A, Campbell H, Rudan I, et al., Lancet Diarrhoea and Pneumonia Interventions Study Group. Interventions to address deaths from childhood pneumonia and diarrhoea equitably: what works and at what cost? Lancet [Internet]. 2013 [citado em 02 maio 2018]; 381(9875):1417-29. Disponível em: https://www.sciencedirect.com/science/arti cle/pii/S0140673613606480?via\%3Dihub. doi: https://doi.org/10.1016/S01406736(13)60648-0.

RECEBIDO: 29/08/18

APROVADO: 08/05/19

PUBLICADO: 07/2019 